\title{
Speciation analysis in healthcare
}

\section{H. Goenaga-Infante}

Published online: 15 December 2010

(C) Springer-Verlag 2010

The development of speciation methods enabling quantification and identification of elemental species has largely been driven by the need to differentiate between species of the same element which may be more toxic or less toxic, or essential to nutrition, often depending on the concentration. Examples of relevance to healthcare include inorganic arsenic, arsenobetaine and arsenocholine; chromium (VI) and chromium (III) and methylmercury and inorganic mercury in a variety of matrices such as food/supplements, biological tissues and clinical and environmental samples. Regulators and legislators are increasingly aware that the toxicity, environmental impact and health benefits of certain trace elements are more dependent on the amount and identity of their chemical forms than on the total concentration. However, most existing regulations are based on total element amount, although a few refer to element species. This is, to a large extent, owing to the limited availability of toxicological data, which demands 'fit-for-purpose' speciation methods. Such methods will become invaluable tools to support emerging regulation and human risk assessment.

For speciation analysis applied to healthcare, approaches combining a high-resolution separation method, either offline or online, with element-specific detectors have been used for many years. However, it was not until the late 1980s that speciation researchers could have access to the detection power needed to measure low parts-per-billion

Published in the special issue Speciation Analysis in Healthcare with Guest Editor Heidi Goenaga Infante.

H. Goenaga-Infante $(\bowtie)$

LGC Limited,

Queens Road,

Teddington, Middlesex TW11OLY, UK

e-mail: heidi.goenaga-infante@lgc.co.uk and parts-per-trillion levels of elemental species in environmental, food and bioclinical samples. Since then, highperformance liquid chromatography-inductively coupled plasma (ICP)-mass spectrometry (MS) techniques have become the centrepiece of most elemental speciation work in this area. Also, the multielement and multi-isotope detection capability of ICP-MS enabled for the first time monitoring of the degree of species transformation (through isotope ratio measurements), which is a challenge associated with most sample preparation procedures. It was mainly in the 1990s when Lobinski and other speciation experts stressed the need to use the combination of elemental and molecular MS and other complementary techniques for elemental species identification over previously reported retention time identification by ICP-MS detection. Today, speciation scientists are more aware of the potential and challenges of this combined approach and, especially, of the power of accurate mass measurement and have, therefore, used it successfully in many applications of speciation analysis to healthcare. Relevant examples include the identification of multiple selenium metabolites of relevance to cancer research and nutrition in food/ supplements, cancer cell lines and biological fluids and tissues, the identification of organoarsenic species (e.g. arsenosugars and arsenolipids) in food and arsenosugars and methylated arsenic species in urine.

The introduction of new concepts, technologies and needs in modern clinical chemistry, such as understanding the role of metalloproteins in health and disease or the impact of metallodrugs in disease diagnosis or treatment, has led to major progress in healthcare elemental speciation but also to an increased number of analytical challenges. The possibility of measuring metal-to-sulfur ratios using interference-reducing ICP-MS opened a new door to the quantification and characterisation of metalloproteins and 
metalloenzymes (e.g. superoxide dismutase) at nanogramper-gram levels in clinical samples. The selectivity and detection power that ICP-MS offers to today's phosphorus speciation research has also been exploited for the determination of protein phosphorylation degree and for quantitation of DNA adduct formation with anticancer drugs (e.g. platinum drugs) as a tool for monitoring the drug efficacy in cancer treatment. More recently, isotope dilution MS (IDMS) methods (e.g. species-specific and postcolumn IDMS) and isotopically enriched species have been developed and used for the quantification of heteroatom-tagged species, including metalloproteins (e.g. iron transferrin and selenoproteins), in clinical applications.

From the foregoing account, great advances have been achieved but further developments are required, especially if 'speciated' certified reference materials are to be produced for measurement validation and quality control in the healthcare area. One problem is the lack of accuracy and traceability of the overall analytical process for most methods in common use. Therefore, traceable standards and accurate reference methods (e.g. IDMS methods) are needed to provide field laboratories with reliable standards and reference materials and to provide independent reference values for proficiency testing schemes related to healthcare. The use of multistep sample preparation or preconcentration procedures is often needed to improve selectivity and, therefore, sample-to-noise ratio for the characterisation of elemental species in complex samples by electrospray ionization (ESI) MS/MS. However, such methods often lead to species transformation. Therefore, advances are urgently needed in the simplification and efficiency of sample preparation methods, which crucially do not change the nature of the elemental species. For ultratrace species characterisation in limited samples (e.g. small cell populations), further development of selective chromatographic methods for micro/nanosamples compatible with both ESI and ICP is still required. Moreover, enhancement of the detection capabilities of new gener- ations of organic mass spectrometers, especially those able to perform accurate mass measurements, is highly desirable. Since toxicity also depends on size, the development and validation of size-based speciation methods will be invaluable not only for future nanotoxicity studies but also for monitoring food and environmental safety. Finally, work across different disciplines and the combination of speciation data with data from biomeasurements will continue to be essential for understanding the healthcare implications of the speciation results.

This special issue comprises a review and a feature article, four original papers and a technical note. Together, these highlight the complexity and role of speciation measurements in providing information relevant to food and environmental safety and compound-specific metabolism. The articles cover a range of elemental species of different types (e.g. stable arsenosugars and labile manganese complexes) and samples of different complexity (e.g. urine, welding aerosols and cells). It is hoped that this collection of excellent papers will help to broaden the horizons for developments in this field. I would like to thank all the authors for their contributions and hope you enjoy reading them.

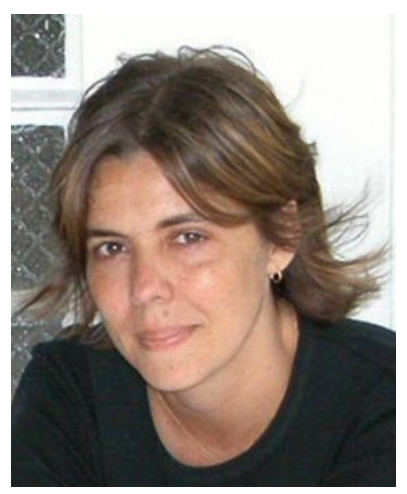

Heidi Goenaga-Infante is currently Principal Scientist for the Chemical Measurement and Calibration Team at LGC Limited in Teddington, UK. Her research interests lie in trace element speciation analysis, metallomics research, combined use of elemental and molecular mass spectrometry, size-based element fractionation and the characterisation of 'speciated' reference materials and standards. She is a member of the International Advisory Board of the journals Analytical and Bioanalytical Chemistry and Metallomics. 